\title{
Generalized Ohm's Law for a Background Plasma in the Presence of Relativistic Charged Particles
}

\author{
M. Sherlock \\ Blackett Laboratory, Imperial College, London SW7 2BZ, United Kingdom
}

(Received 30 July 2009; published 20 May 2010)

\begin{abstract}
A generalized Ohm's law is derived for a system composed of a background magnetohydrodynamic plasma and a lower density relativistic charged-particle distribution. The interpretation of Ohmic electric fields occurring due to force balance breaks down for such a system and instead an approach based on Maxwell's equations along with the particle flux equations is necessary. Three additional terms arise in Ohm's law and each is verified numerically.
\end{abstract}

DOI: 10.1103/PhysRevLett.104.205004

Introduction.-The generalized Ohm's law is important in plasma theory because it allows us to circumvent Maxwell's equations and determine the electric field $\mathbf{E}$ in terms of macroscopic plasma parameters. As such it is central to theories as varied as magnetohydrodynamics and laser-plasma transport. Although the generalized form has been shown to contain at least 13 terms [1], reduced forms are often used by ignoring relatively small terms. Those most commonly employed appear in the example:

$$
\mathbf{E}=-\mathbf{V} \times \mathbf{B}-\frac{\nabla P_{e}}{e n_{e}}+\eta \mathbf{j}
$$

where $\mathbf{B}$ is the magnetic field, $\mathbf{V}$ is the bulk plasma velocity, $e$ is the electron charge, $n_{e}$ is the electron number density, $P_{e}$ is the electron thermal pressure, $\eta$ is the resistivity, and $\mathbf{j}$ is the thermal current density. The first, second, and third terms on the right-hand side (rhs) of (1) are often dominant in the areas of ideal MHD, long-pulse laser-plasma interactions and charged-particle transport in a dense background plasma (respectively). In this Letter we address the question of how collisionless, energetic particles can induce electric fields as they stream through a background MHD plasma. Beginning with a two-fluid theory, the background (or cold) electrons satisfy the momentum equation

$$
\frac{\partial \mathbf{p}_{c}}{\partial t}+\boldsymbol{\nabla} \cdot \mathbf{P}_{c}=-e n_{c}\left(\mathbf{E}+\mathbf{v}_{c} \times \mathbf{B}\right)+\mathbf{R}_{c}
$$

while the energetic (or fast) particles satisfy

$$
\frac{\partial \mathbf{p}_{f}}{\partial t}+\boldsymbol{\nabla} \cdot \mathbf{P}_{f}=Q_{f} n_{f}\left(\mathbf{E}+\mathbf{v}_{f} \times \mathbf{B}\right),
$$

where $\mathbf{p}$ is the momentum density, $\mathbf{P}$ is the pressure tensor, $\mathbf{v}$ is the average velocity, $Q$ is the charge on each particle, $\mathbf{R}$ is the rate of change of momentum density due to collisions, and we have used subscripts $c$ and $f$ to denote cold and fast components, respectively. We have ignored $\mathbf{R}_{f}$ because, while it may be important for fast-particle transport, in practice this usually occurs when it is simultaneously not important in Ohm's law. The generalized
PACS numbers: 52.30.Cv, 52.27.Ny, 52.30.Ex, 52.57.Kk

Ohm's law (in a single-electron-component plasma) is often expressed as a simple rearrangement of the cold electron equation of motion (2) by ignoring the inertial term $\left(\partial \mathbf{p}_{c} / \partial t\right)$

$$
\mathbf{E}=\frac{1}{e n_{c}}\left\{-\boldsymbol{\nabla} \cdot \mathbf{P}_{c}+\mathbf{j}_{c} \times \mathbf{B}+\mathbf{R}_{c}\right\}
$$

in which $\mathbf{j}_{c}=-e n_{c} \mathbf{v}_{c}$. The time scale over which the inertial term can be ignored depends on the system: in a highly magnetized plasma it is assumed that oscillations at the electron cyclotron frequency can be ignored because they cause the inertial term to average to zero, in which case electrons simply undergo drift; in a collisional plasma, collisions limit the net force after a few collision times. Since we are interested in a fast population which is neither highly collisional nor highly magnetized over time scales of interest, we cannot neglect the inertial term in (3) in comparison to the others. Thus it is not possible to simply sum equations (2) and (3) and ignore both inertial terms on the basis of time-scale arguments. An alternative strategy [2] is to continue using Eq. (4) - after all we do not intuitively expect the presence of the fast component to alter the physical time scales associated with the cold background-for example if the background is collisional in the absence of a fast component then it should remain collisional in the presence of a fast component; all that is necessary is to provide a working expression for $\mathbf{j}_{c}$, which is easily given by current balance $\mathbf{j}_{c}=-\mathbf{j}_{f}$. However, this approach neglects the fact that while the physical time scales associated with the various terms in the cold background equation may remain unchanged, the current induced by the fast component must somehow be reflected in the electric field. As we shall see, these effects should enter Eq. (4) through the omitted inertial term which may no longer be negligible because the cold background is forced to maintain quasineutrality by responding on fast-particle time scales. The aim of this Letter is to derive an Ohm's law valid in the presence of relativistic streaming particles, taking into account a proper method for removing the inertial terms (which makes practical use of the equation possible). 
While not explicitly seeking a generalized Ohm's law, Cox and Bennett [3] derived expressions for the electromagnetic potentials in fixed frequency regimes for a cold relativistic beam of fixed profile passing through a background plasma. The Ohm's law for an electron-positron plasma has been derived by Gedalin [4] in limits appropriate for pair plasmas. Kandus [5] has derived a generalrelativistic Ohm's law which retains the inertial terms. While inertial terms are important for complete generality, on a practical basis they are highly undesirable because they require further equations to be specified in order to solve for them.

We are primarily motivated by the large number of experiments in the field of intense laser-solid interactions (see, e.g., [6-8]) though our resulting Ohm's law may have applications in fast-ignition [9], astrophysics [10], magnetic reconnection [11], pulsed-power devices [12] and fast-particle transport in magnetic-confinement devices [13]. In each of these topics, the electron or ion distribution functions frequently contain a fast component which can be treated as a separate kinetic species propagating through the bulk "background" plasma.

Our starting point is the Ampère-Maxwell law

$$
\frac{\partial \mathbf{E}}{\partial t}=c^{2} \nabla \times \mathbf{B}-\frac{\mathbf{j}}{\boldsymbol{\epsilon}_{0}},
$$

which immediately tells us that (in the absence of a magnetic field) the electric field will grow if net currents are not suppressed-i.e., the electric field acts to suppress a net current flow (and not necessarily momentum flow). Put another way, plasma quasineutrality is maintained by ensuring the electrons and ions copropagate rather than gain the same momentum. This leads us to write equations for the evolution of the background and fast-particle current densities by integrating over the kinetic equations (see [14] as a guide to this analysis):

$$
\begin{gathered}
\frac{\partial \mathbf{j}_{c}}{\partial t}-\frac{e}{m_{e}} \boldsymbol{\nabla} \cdot \mathbf{P}_{c}=\frac{e^{2} n_{c}}{m_{e}} \mathbf{E}-\frac{e}{m_{e}} \mathbf{j}_{c} \times \mathbf{B}-\nu_{\mathrm{ci}} \mathbf{j}_{c}, \\
\frac{\partial \mathbf{j}_{f}}{\partial t}+\boldsymbol{\nabla} \cdot\left\langle\mathbf{j}_{f} \mathbf{v}_{f}\right\rangle=\frac{Q_{f}^{2} n_{f}}{m_{f}} \mathbf{E} \cdot\langle\mathbf{C}\rangle+\frac{Q_{f}}{m_{f}}\left\langle\mathbf{C} \cdot \mathbf{j}_{f} \times \mathbf{B}\right\rangle,
\end{gathered}
$$

where $m_{f}$ is the mass of each fast particle, $\mathbf{C} \equiv \partial \mathbf{v}_{f} / \partial \mathbf{U}_{f}$ is a symmetric tensor in the fast-particle relativistic threevelocity $\mathbf{U}=\gamma_{f} \mathbf{v}_{f}, \nu_{\mathrm{ci}}$ is the cold-electron-ion collision frequency, the brackets \langle\rangle denote an average over the fastparticle distribution function, and we have assumed the background electrons are nonrelativistic. Note that in the term $\nu_{\text {ci }} \mathbf{j}_{c}$ of Eq. (6) (and all following collision terms) the cold current $\mathbf{j}_{c}$ refers to that calculated in the ion rest frame. Although other collision terms are present (see, e.g., [15]), we have reduced $\mathbf{R}_{c}$ to the resistivity for simplicity. Assuming the background ions do not significantly contribute to the rate of change of total current density (due to their relatively large mass), $\partial \mathbf{j} / \partial t=$ $\partial\left(\mathbf{j}_{c}+\mathbf{j}_{f}\right) / \partial t$. Using this, Eqs. (5)-(7) can be combined with Faraday's law to yield the wave equation

$$
\frac{\partial \mathbf{j}_{c}+\mathbf{j}_{f}}{\partial t}=\epsilon_{0} \frac{\partial^{2} \mathbf{E}}{\partial t^{2}}-\epsilon_{0} c^{2} \boldsymbol{\nabla} \times \boldsymbol{\nabla} \times \mathbf{E} .
$$

Next we make a number of limiting assumptions:

$$
n_{c} \gg n_{f} \frac{m_{e}}{m_{f}} \frac{Q_{f}^{2}}{e^{2}}|\mathbf{C}|, \quad \tau \gg \frac{1}{\omega_{p c}}, \quad L \gg \frac{c}{\omega_{p c}},
$$

where $\omega_{p c}=\sqrt{e^{2} n_{c} / m_{e} \epsilon_{0}}$ and $\tau$ and $L$ are the characteristic time and length scales associated with the operators $\partial / \partial t$ and $\boldsymbol{\nabla} \times$, respectively. For the case of nonrelativistic fast electrons, the first approximation above simply corresponds to $n_{f} \ll n_{c}$; the second simply states that we are ignoring effects which occur on time scales comparable to the cold-plasma period and the third corresponds to ignoring effects which occur on length scales comparable to the cold-plasma skin depth. Ignoring terms by such order-ofmagnitude arguments is equivalent to Fourier-filtering out high frequency effects from the linearized form of the equations. In general the scales $\tau$ and $L$ should be thought of as applying to a field which has been temporally and spatially averaged over scales long enough to satisfy the assumptions (9) (though this may happen naturally if there is even a small amount of dissipation in the system). With these assumptions in mind, the two equations (6) and (7) can be summed and combined with both (5) and (8) to yield an expression for the field without inertial terms:

$$
\begin{aligned}
\mathbf{E}= & \frac{1}{e n_{c}}\left\{\frac{\boldsymbol{\nabla} \times B}{\mu_{0}}-\mathbf{j}_{f}-\mathbf{j}_{i}\right\} \times \mathbf{B}+\frac{1}{e n_{c}}\left\langle\mathbf{C} \cdot \mathbf{j}_{f} \times \mathbf{B}\right\rangle \\
& -\frac{1}{e n_{c}} \boldsymbol{\nabla} \cdot \mathbf{P}_{c}+\frac{m_{e}}{e^{2} n_{c}} \boldsymbol{\nabla} \cdot\left\langle\mathbf{j}_{f} \mathbf{v}_{f}\right\rangle+\eta \mathbf{j}_{c} .
\end{aligned}
$$

This expression consists of familiar terms in cold electron quantities but contains the three new terms in $\boldsymbol{\nabla} \cdot\left\langle\mathbf{j}_{f} \mathbf{v}_{f}\right\rangle$, $\left\langle\mathbf{C} \cdot \mathbf{j}_{f} \times \mathbf{B}\right\rangle$ and $\mathbf{j}_{f} \times \mathbf{B}$. In the ideal MHD approximation (which corresponds to ignoring thermal and dissipative terms as well as the Hall term $\boldsymbol{\nabla} \times \mathbf{B} \times \mathbf{B} / e n_{c} \mu_{0}$ in Ohm's law), we have

$$
\mathbf{E}=-\frac{1}{e n_{c}}\left\{\mathbf{j}_{f}+\mathbf{j}_{i}\right\} \times \mathbf{B}+\frac{1}{e n_{c}}\left\langle\mathbf{C} \cdot \mathbf{j}_{f} \times \mathbf{B}\right\rangle .
$$

In the nonrelativistic limit, $\left\langle\mathbf{C} \cdot \mathbf{j}_{f}\right\rangle \rightarrow \mathbf{j}_{f}$ and the above reduces to the well-known form $\mathbf{E}=-\mathbf{u}_{i} \times \mathbf{B}$, where $\mathbf{u}_{i}$ is the ion fluid velocity and we assume quasineutrality $\left(Z n_{i}=n_{c}\right)$; in the ultrarelativistic limit, $\left\langle\mathbf{C} \cdot \mathbf{j}_{f}\right\rangle \rightarrow 0$ so instead the above becomes $\mathbf{E}=-\left(\mathbf{j}_{i}+\mathbf{j}_{f}\right) \times \mathbf{B} /$ en $_{c}$. This latter expression would lead to a breakdown of the freezing-in of magnetic flux if $\mathbf{j}_{f} \gg \mathbf{j}_{i}$, in which case the magnetic flux would be advected at velocity $\mathbf{j}_{f} / e n_{c}$, rather than at $\mathbf{u}_{i}$.

In the remainder of this Letter we will show results of simulations which verify the existence of the new terms. The various terms are tested with two different numerical models-each one appropriate to the nature of the term being verified. 
Numerical verification of the magnetic-field terms.-In this section we describe simple simulations which verify the new terms in

$$
\mathbf{E}=\eta \mathbf{j}_{c}+\frac{1}{e n_{c}}\left\langle\mathbf{C} \cdot \mathbf{j}_{f} \times \mathbf{B}\right\rangle-\frac{1}{e n_{c}} \mathbf{j}_{f} \times \mathbf{B},
$$

viz, those in $\left\langle\mathbf{C} \cdot \mathbf{j}_{f} \times \mathbf{B}\right\rangle$ and $\mathbf{j}_{f} \times \mathbf{B}$. Since these terms contain magnetic field but no spatial gradients, we can choose a homogeneous situation and make use of zerodimensional particle simulation. Particle simulation is particularly well suited to the magnetic-field terms because there are existing techniques for accurately integrating the equations of motion for particles subject to a Lorentz force. While Eqs. (2) and (3) refer to averaged quantities, the same principles apply, and we solve them along with the Ampère-Maxwell law in a homogeneous magnetic field by using the well-known second-order Boris algorithm. The beam-to-background density ratio is $1 / 1000$ and the fast particles are chosen to be electrons. We ensure the coldplasma period is temporally resolved and assume a fixed background of ions. The nonrelativistic limit of Eq. (12) is trivial because it involves only the resistivity term. Conversely, the ultrarelativistic limit does not contain significant contributions from the term in $\boldsymbol{\nabla} \cdot\left\langle\mathbf{j}_{f} \mathbf{v}_{f}\right\rangle$. We therefore show $E_{x}(t)$ in the more demanding intermediate regime for a fast-particle relativistic $\gamma=1.5$ (with initial momentum in the $x$ direction), to illustrate the contribution from all terms. In Fig. 1, each term is labeled according to its order of appearance on the rhs of Eq. (12). The fast particles are initially smoothly accelerated up to their peak energy and then a magnetic-field is slowly switched on (but reaches a steady value), as indicated by the labels in the figure. It is clear that all terms are important. For the curve labeled Ohm's law in the figure, we also included a term to

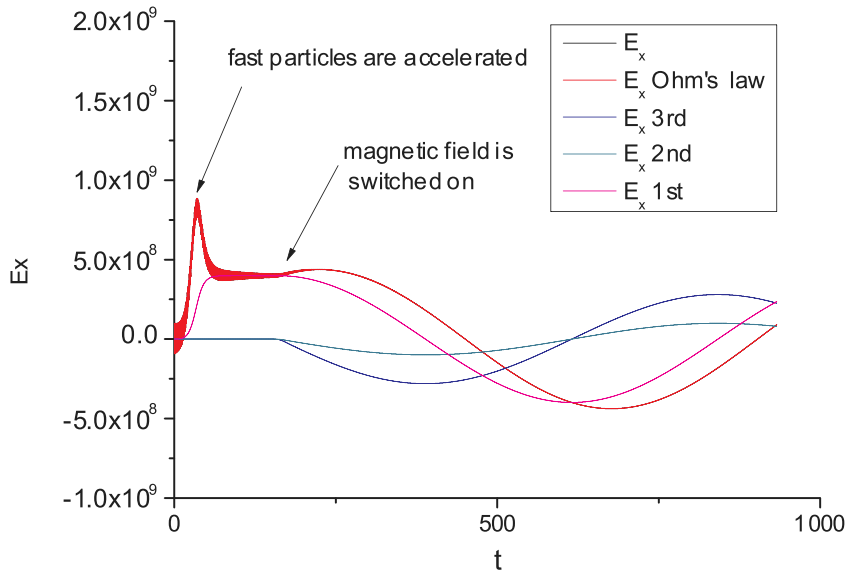

FIG. 1 (color online). The electric field as found in simulations which solve Maxwell's equations and the particle equations of motion. The fast particles undergo an initial acceleration period up to a relativistic $\gamma=1.5$, shortly after which a magnetic field is applied which induces a harmonic electric field. Also shown are the various contributions to the field from Ohm's law labeled according to their order of appearance on the rhs of Eq. (12). account for the initial applied particle acceleration period (but this is ignorable once the applied force ceases). Note that our expression for the field agrees so closely with the actual field it is difficult to distinguish the two curves in Fig. 1. For reference, we give the field arising from the $x$ component of the term $\left\langle\mathbf{C} \cdot \mathbf{j}_{f} \times \mathbf{B}\right\rangle$ in terms of the fastparticle momentum $\left(q_{f x, y}\right)$ :

$$
E_{x}=\frac{1}{e n_{c}}\left\{\frac{1}{\gamma_{f}}\left(1-\frac{q_{f x}^{2}}{(m c)^{2} \gamma_{f}^{2}}\right) j_{y}+\frac{q_{f x} q_{f y}}{(m c)^{2} \gamma_{f}^{3}} j_{x}\right\} B_{z} .
$$

Intense laser-plasma interactions.-Taking an alternative limit of Eq. (10) relevant to laser-solid interactions in the relativistic regime [16], where it is often the case that Ohm's law need not contain the pressure term and the magnetic-field terms are negligible (provided $B$ is of the order of $10^{2} \mathrm{~T}$ ), we arrive at

$$
\mathbf{E}=\frac{m_{e}}{e^{2} n_{c}} \boldsymbol{\nabla} \cdot\left\langle\mathbf{j}_{f} \mathbf{v}_{f}\right\rangle+\eta \mathbf{j}_{c} .
$$

Assuming current balance $\left(\mathbf{j}_{c}=-\mathbf{j}_{f}\right)$, the first term on the rhs of Eq. (14) dominates wherever $L / c<\tau_{\mathrm{ci}}$, where $L$ is the characteristic scale length of the fast-particle current and $\tau_{\mathrm{ci}}$ is the cold-plasma electron-ion collision time. In intense laser-solid interactions, relativistic electrons are accelerated into the target in short pulses with a scale length $L \approx c \tau_{0} / 4$, where $\tau_{0}$ is the period of the electromagnetic wave. In this case the term $m_{e} / e^{2} n_{c} \boldsymbol{\nabla} \cdot\left\langle\mathbf{j}_{f} \mathbf{v}_{f}\right\rangle$ dominates if $\tau_{0}<4 \tau_{\text {ci }}$. This occurs in a sufficiently low density or high temperature plasma, such as that near the front surface of the interaction. We have plotted the relative magnitude $\tau_{\mathrm{ci}} /\left(\tau_{0} / 4\right)$ in Fig. 2 as a function of background electron temperature, for various densities (normalized to nonrelativistic critical density of $1 \mu \mathrm{m}$ light).

In order to demonstrate the validity of the first term in Eq. (14), we have performed direct kinetic simulations of a laser-plasma interaction in $1 \mathrm{D}$ with the simulation code FIDO. FIDO solves the relativistic Vlasov-Fokker-Planck equation and Maxwell's equations together and we fully resolve the background plasma period. Momentum space is described on a spherical computational grid, which facil-

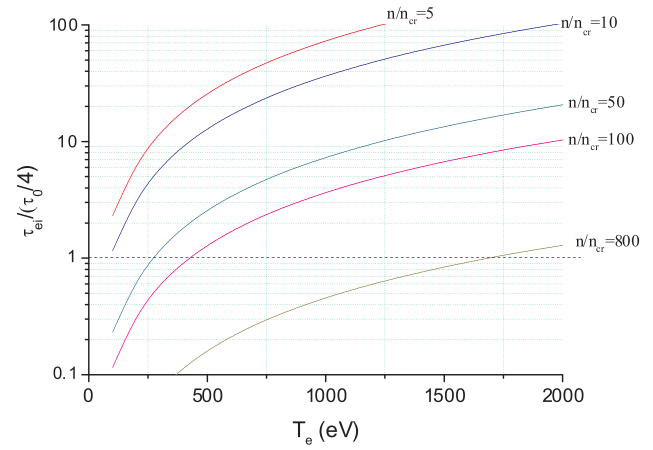

FIG. 2 (color online). The relative magnitude of the two terms in Eq. (14), equivalent to the ratio $\tau_{\text {ci }} /\left(\tau_{0} / 4\right)$ as a function of background electron temperature, for a range of densities (normalized to the nonrelativistic critical density of $1 \mu \mathrm{m}$ light). 


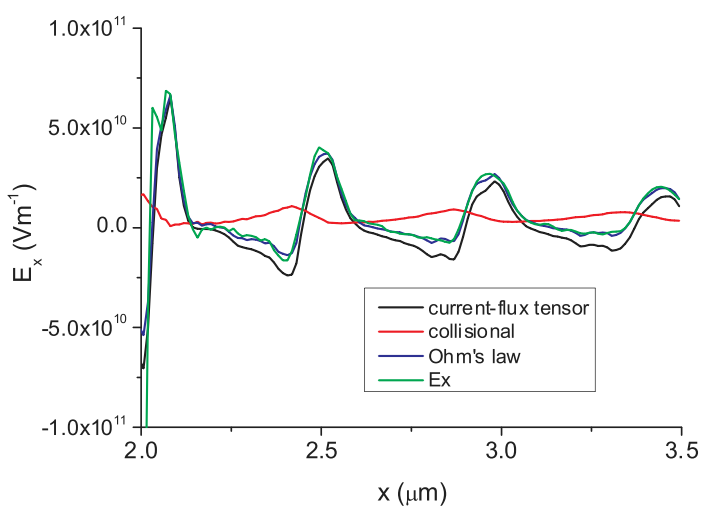

FIG. 3 (color online). The longitudinal electric field set up as a result of fast-electron pulses streaming from left to right, inside a solid-Al plasma. Also shown is the field given by the Ohm's law Eq. (14) and its contributions from the first (current-flux tensor) and second (collisional) terms. The data are shown at $t=30 \mathrm{fs}$. Note that the spatial range begins inside the dense target.

itates a rapid electron-ion angular-scattering algorithm and preserves rotational symmetry of the distribution function under the action of the $\mathbf{v} \times \mathbf{B}$ force. We prefer a finitedifference approach to solving the equations over particle simulation in order to minimize noise and arrive at a "clean" instantaneous longitudinal field $E_{x}$. The computational cost of such simulations is, however, high, due to the Courant stability condition in momentum space (since we resolve an initial $1 \mathrm{keV}$ background distribution as well as multi-MeV fast electrons within the same distribution). Shown in Fig. 3 is the longitudinal electric field during a simulation in which a laser, at intensity $5 \times 10^{19} \mathrm{~W} \mathrm{~cm}^{-2}$ and wavelength $1 \mu \mathrm{m}$, is incident from the left-hand boundary. The solidAl plasma initially has a relatively sharp density scale length (5\% of the laser wavelength) and the laser intensity is increased linearly in time over the first four laser cycles and is thereafter constant. Pulses of relativistic electrons are produced by the well-known $\mathbf{j} \times$ B mechanism [17], which stream into the target and induce an electric field in order to maintain quasineutrality. Also shown is the field given by Ohm's law [Eq. (14)] and its contributions from the first ("current-flux tensor") and second ("collisional") terms. Our example clearly demonstrates that the current-flux tensor associated with the relativistic electron bunches gives rise to an electric field which exceeds the resistively induced field by over an order of magnitude. These fields will lead to enhanced background heating (via $\mathbf{j} \cdot \mathbf{E}$ ) and in two dimensions magnetic field generation which tends to increase fastelectron collimation. They are particularly important in long scale-length plasma, such as that created at the front of solid targets by the laser prepulse.

On the subject of solid targets, it should be noted that this work is only directly applicable to metal targets. In the case of dielectrics the need for ionization of the background material substantially changes the situation [18], and an ionization current should be added to the rhs of
Eq. (6). For the case of electron beams in foam or gas targets [19], we stress that the conditions (9) must apply, in particular, the beam density must be much lower than the background density. When this is not the case, the resistivity must be modified because the isotropic component of the background distribution function becomes strongly non-Maxwellian [20].

Finally, we show how the bulk background flow is modified due to the presence of fast particles. By defining the background plasma momentum density as $\mathbf{p}_{p}=\mathbf{p}_{i}+$ $\mathbf{p}_{c}$ and again using the assumptions (9) with Ampère's law, we arrive at

$$
\frac{\partial \mathbf{p}_{p}}{\partial t}+\boldsymbol{\nabla} \cdot\left(\mathbf{P}_{i}+\mathbf{P}_{c}\right)=\left(\frac{\boldsymbol{\nabla} \times \mathbf{B}}{\mu_{0}}-\mathbf{j}_{f}\right) \times \mathbf{B},
$$

which is just the standard MHD equation of motion (i.e., in the absence of a fast component) augmented by the reactive force $-\mathbf{j}_{f} \times \mathbf{B}$, as inferred by Bell [10].

In summary, we have derived a generalized Ohm's law [Eq. (10)] for the electric field in a background MHD plasma in the presence of relativistic charged particles by ensuring quasineutrality via the Ampère-Maxwell law. The limits of validity of the model are outlined in Eqs. (9). The fast particles give rise to three new field-generation terms, each of which has been verified by numerical simulation.

[1] M. G. Haines, Can. J. Phys. 64, 912 (1986).

[2] X. Zha, Y. Wang, and S. Han, High Energy Density Phys. 4, 103 (2008).

[3] J. L. Cox and W. H. Bennett, Phys. Fluids 13, 182 (1970).

[4] M. Gedalin, Phys. Rev. Lett. 76, 3340 (1996).

[5] A. Kandus and C. G. Tsagas, Mon. Not. R. Astron. Soc. 385, 883 (2008).

[6] M. Borghesi et al., Phys. Rev. Lett. 83, 4309 (1999).

[7] D. Batani et al., Phys. Rev. E 65, 066409 (2002).

[8] P. A. Norreys et al., Plasma Phys. Controlled Fusion 48, L11 (2006).

[9] M. Tabak, J. Hammer, M. E. Glinsky, W. L. Kruer, S. C. Wilks, J. Woodworth, E. M. Campbell, M.D. Perry, and R. J. Mason, Phys. Plasmas 1, 1626 (1994) [http:// link.aip.org/link/?PHP/1/1626/1].

[10] A. R. Bell, Mon. Not. R. Astron. Soc. 353, 550 (2004).

[11] J. F. Drake, M. A. Shay, W. Thongthai, and M. Swisdak, Phys. Rev. Lett. 94, 095001 (2005).

[12] N. R. Pereira and J. Davis, J. Appl. Phys. 64, R1 (1988).

[13] S. D. Pinches et al., Plasma Phys. Controlled Fusion 46, B187 (2004).

[14] D. Mosher, Phys. Fluids 18, 846 (1975).

[15] E. M. Epperlein and M. G. Haines, Phys. Fluids 29, 1029 (1986).

[16] A. R. Bell et al., Plasma Phys. Controlled Fusion 39, 653 (1997).

[17] W. L. Kruer and K. Estabrook, Phys. Fluids 28, 430 (1985).

[18] V. T. Tikhonchuk, Phys. Plasmas 9, 1416 (2002).

[19] D. Batani et al., Phys. Rev. Lett. 94, 055004 (2005).

[20] M. Sherlock et al., Phys. Plasmas 14, 102708 (2007). 